\title{
Fuzzy Approach to Regulate S-type Biological Systems
}

\author{
Shinq-Jen Wu \\ Department of Electrical Engineering Da-Yeh University, \\ 168 University Rd., Dacun \\ Changhua 51591, Taiwan, R.O.C
}

\begin{abstract}
It is important to regulate biological systems to return to nominal steady states such that systems are able to maintain normal functions.S-type biological systems(S-systems) are described as power-law-based differential equations which are able to shownetinteractive strength between constitutes and a result, S-system becomes the most potential model for largescale systems. Biological systems always possess a lot of uncertainties and noises. Fuzzy sets and models are able to describe, recognize and manipulate data that are vague and lack certainty.However, biological systems are different from electromechanical systems thatallow various types of timevarying signalsas system inputs. Therefore, step functions are used as fuzzy outputs to denote constant concentration and the firing strength of each fuzzy rule is the blending or allocating ratio.A cascade pathway is concerned andan exponentially decaying model is used to describe the functional degradation phenomenon. Dry-lab experiments are carried out in five different situations. Simulation results show that the proposed seven-rule fuzzy logic controllers are able to find out the nominal values of independent variables and force systems to return to their nominal steady states. The larger the nominal values are the longer the time to reach targets.
\end{abstract}

\section{Keywords}

Fuzzy logic control, systems biology, computational biology

\section{INTRODUCTION}

Generalized Michaelis-Menten kineticsdescribes physically interactive kinetics between constitutes (proteins, genes and metabolites). S-systems(S-type biological systems) in Eq. (1) show net interactive strength between constitutes.

$$
\begin{aligned}
& \dot{x}_{i}=v_{i}^{+}-v_{i}^{-} \\
& =\alpha_{i} \prod_{j=1}^{n+m} x_{j}^{g_{i j}}-\beta_{i} \prod_{j=1}^{n+m} x_{j}^{h_{i j}}, i=1, \ldots n
\end{aligned}
$$

where $g_{i j}$ and $h_{i j}$ denote the net interactive strength from $x_{j}$ on $x_{i}, \alpha$ and $\beta_{i}$ are the rate constants. The $x_{i}, i=1, \ldots n$ are dependent variables and $x_{n+1}, \ldots x_{n+m}$ are independent variables, the values of which remains constant during a period of an experiment.These two well-known biological systems are bothbased on biochemistry system theory and described as highly nonlinear differential equations. The modelling of generalized Michaelis-Menten systems is a bottom-up process. System are gradually constructed from small systems [1] to medium-sized systems [2] and then expanded to large systems [3]. Parametersare estimated through doing experiments over and over again and a large amount of experimentaldata are used. The modelling of Ssystems is a top-down process and parameters are estimated through computational approaches. Good generalization properties let S-system become the most potential model for large-scale systems. Liu and coworkers used the S-system to describe p53 signaling pathway mechanism [4]. S-system modelling is a multi-objective multi-constraints optimization problem. Various intelligently computational technologies wererecently developed to achieve S-system modelling [5$11]$.

There exist too many uncertainties and noises in a biological system. Luo and An reviewed fuzzy theory in four kinds of biomedical science (device control, biological control, classificationand pattern recognition, and prediction and association) [12].Komlyama and coworkers did a deep-review on DNA nano architectonics and cell-macromolecular nano architectonics [13].They emphasized that many biological mechanisms (for example, hydrophobic effects and electrostatic interactions) exist rather fuzzy molecular interactions.So, exact or optimal methods have considerable limitations in this fields.Fuzzy logic comes from an observation that the thinking of people is always based on imprecise and non-numerical information. Fuzzy sets and models are able to describe, recognize and manipulate data that are vague and lack certainty.The use of linguistic variables and imprecise relationship let fuzzy set theory have great potential in analyzing biological data, modeling biological systemsand solving a wide range of biological problems. Abyad and coworkers used the T-S fuzzy model to describe a biomass growth process, a growing population of microorganisms through substrate consumption [14]. Bordon et al. used fuzzy logic to describe a three-gene repressilator with unknown kinetic data [15].Liu and coworkers reviewed three kinds of fuzzy Petri nets for biological system modelling and discussed modelling capacities and applications [16].They integrated continuous Petri nets with fuzzy inference system and proposed a workflow for modelling and analyzing biological systems [17]. Zhu and coworkers combined fuzzy neural network inverse system methods and decupling control technologies to achieve the control of marine alkaline protease MP (a kind of extracellular enzyme) [18].Adaptive neuralfuzzy modelingtechnique was previously proposed to identify biological systems and discussed the scalability of the proposed method [19].

Biological systems always possess a good regulation mechanism to maintain a system within certain limits. When systems are in a bad situation (for example, modellable experimental variables denoted as independent variables decay with time) the regulation mechanism fails to work and systems are not able to return to nominal states(steady states). In this study, fuzzy logic controllersare used to regulate biological systemsback totheir nominal states.

\section{METHODS}

When individuals getsickthe dynamic behavior of underlying systemsgoes far from their nominal states (steady states). Systems can not automatically return back to their nominal states and additional treatments are required. 


\section{Exponentially decaying model}

The degradation of body functional changes the environment of biological systems and makes system loss the regulation ability. Whenmedicinesare administered intravenouslythe concentration decreasesas time goes due to liver metabolism and kidney filtration (clearance of liver and kidneys [20]).In thesesituations, systeminputs(independent variables, modellable experimental variables) cannot remain at a constant value and will continually decreaseas time goes (time-varying input). In this study, an exponential function $r=r_{0} e^{a t}$ is used to describe the decaying phenomenon, where $r_{0}$ is a starting value of concentration of independent variables, $\mathrm{e}$ is the Euler's constantand $a$ is a negative constant that determines the rate of decay [21]. (The exponentially decaying model isalways used in biology and radioactive isotopes to estimatethe half-life, the time for a substance to exponentially decay to half of its original quantity.)The exponentially decaying model is expressed as block diagrams in the Simulink environment, as shown in Fig. 1 .

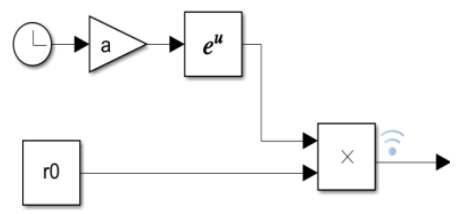

Fig 1:Exponential decay model blocks

Prototype of biological fuzzy control

When system statesare out of suitable living environmentsfuzzy control is used to drivesystemsback to nominal states. However, biological systems are different from electromechanical systemsthatallow various types of time-varying signalsas system inputs (impulse, exponentially signals...)A fuzzy model prototype is proposed: Eq. (2) describes the ith rule of the used fuzzy controllerwhere $x_{i}$ and $y$, respectively, denote the linguistically input and output variables, $A_{1 i}, B_{1 i}$ are fuzzy sets and the fuzzy set $C_{i}$ denotes astep function. The firing strengthof each fuzzy rule (denoting the degree to which the rule matches the inputs)isconsidered as a blending or allocating ratio ofthe fuzzy sets $C_{i}, i=1, \ldots n$. The fuzzy logic block in Simulink toolbox, as shown in Fig. 2, is then used to build up the fuzzy controller.

$$
\boldsymbol{R}^{i} \text {. If } x_{1} \text { is } A_{1 i} \text { and } x_{2} \text { is } B_{1 i} \text {, then } y=C_{i}, i=1, \ldots n \text {. }
$$

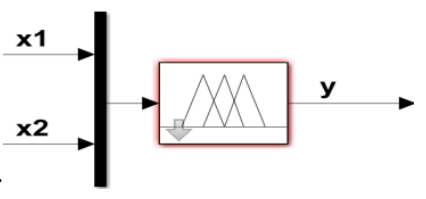

Fig 2:Fuzzy logic controller blocks

\section{Simulink-based behavior \\ dynamic}

Simulink is a toolbox of Matlab (MATrix LABoratory, a software developed by The MathWorks Company). In the Matlab/Simulink software environment, locally dynamic behavior is treated as a block. The dynamic behavior of asystemis obtained through a connection of blocks.Blocks arefurther grouped into a subsystem (a module) which is composed a set of connected blocks. In this way,a large-scale system can be shown as a concise diagram. In addition to achieve theprediction of dynamic behaviorof biological systems in various modellable experimental environments (denoted as input signals), the Simulink environment further provides peoplea more conducive way on the modification and maintenance of individual modules and the modification of interaction between modules.

\section{RESULTS AND DISCUSSION}

A small S-type system is considered to describe the proposed fuzzy approach clearly. Figure 3 is a cascade pathway [22] The source $x_{4}$ is an independent variable which generates midproduct $x_{1}$. The generation reaction is inhibited by both $x_{2}$ and $x_{3}$. The $x_{1}$ induces the generation of mid-product $x_{2}$ which further induces the generation of the final product $x_{3}$.

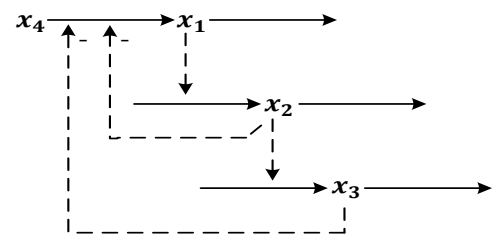

Fig 3: Cascade pathways [22]

The corresponding S-type biological systems (called Ssystems in biology fields) is

$$
\begin{gathered}
\dot{x}_{1}=10 x_{2}^{-0.1} x_{3}^{-0.05} x_{4}-5 x_{1}^{0.5}, \\
\dot{x}_{2}=2 x_{1}^{0.5}-1.44 x_{2}^{0.5}, \\
\dot{x}_{3}=3 x_{2}^{0.5}-7.2 x^{0.5} .
\end{gathered}
$$

The values of exponent order and rate constants are cited from Tsai and Wang's paper [22]. In normal situations, the concentration of the independent variable $x_{4}$ always keeps constant throughoutan experiment.Asituation is considered, in whichsystems experience functional degradationthat leads to the value of source constant $x_{4}$ decreasing from time to time. The exponentially decaying model in Sec. 2 is used to describe this phenomenon: $x_{4}=r_{0} e^{a t}$ were $r_{0}$ is the nominal value of $x_{4}$.

\section{Nominal steady state estimation (target states)}

At equilibrium the net flux $\bar{v}_{i}^{+}=\bar{v}_{i}^{-}$. (The upper bar denotes equilibrium).Equation (1) becomes an algebraic equation in Eq. (4)through setting $y_{i}=\ln x_{i}, g h_{i j}=g_{i j}-h_{i j}, \alpha \beta_{i}=$ $\ln \left(\frac{\beta_{i}}{\alpha_{i}}\right)$ and letting matrices $b=\left[\alpha \beta_{i}\right]_{i=1 \ldots n}, \bar{Y}_{d}=$ $\left[\bar{y}_{j}\right]_{j=1 \ldots n}$ and

$Y_{I}=\left[y_{j}\right]_{j=n+1 \ldots n+m}$, and $A_{I}=\left[g h_{i j}\right]_{i=1 \ldots n, j=n+1 \ldots n+m}$ and $A_{d}=\left[g h_{i j}\right]_{i=1 \ldots n, j=1 \ldots n}$.

$$
A_{d} \bar{Y}_{d}+A_{I} Y_{I}=b
$$

The steady state values is estimated: $\bar{Y}_{d}=A_{d}^{\dagger} \cdot b-\left(A_{d}^{\dagger} A_{I}\right)$. $Y_{I}$, where $A_{d}^{\dagger}$ is the inverse or the pseudoinverse of $A_{d}$.In this case, $\quad n=3, m=1, A_{d}=\left[\begin{array}{ccc}-0.5 & -0.1 & -0.05 \\ 0.5 & -0.5 & 0 \\ 0 & 0.5 & -0.5\end{array}\right], A_{I}=$ $\left[\begin{array}{l}1 \\ 0 \\ 0\end{array}\right]$ and $b=\left[\begin{array}{c}-0.6931 \\ -0.3285 \\ 0.8755\end{array}\right]$. The steady states $\bar{x}_{i}, i=1, \ldots 3$ depend on the independent variables $x_{4}$. When the independent variables $x_{4}$ cannot keep at the constant value $r_{0}$ the system cannot reach a equilibrium point.

\section{Fuzzy logic control}

Fuzzy controller is developed to regulate the system back to 
the equilibrium state $X_{S}\left(\left[\bar{x}_{1}, \bar{x}_{2}, \bar{x}_{3}\right]^{T}\right)$ in the case of the independent variable $x_{4}$ exponentially decaying with time $\left(x_{4}=r_{0} e^{-0.2 t}\right)$.In order to solve this issue the independent variable $x_{4}$ is treated as a system input. The problem becomes a regulator problem.Afuzzy controller is then constructed toforceall of theerrors, $e_{i}=x_{i}-\bar{x}_{i}, i=1, \ldots 3$, to reach zero. Since the concentration of $x_{1}$ and $x_{3}$ is related to the concentration of $x_{2}$. For simplification the $x_{2}$ is used as a target to follow: $e_{2}=x_{2}-\bar{x}_{2}$. Table 1 and Eq. 5 show the sevenrules ofthe proposed fuzzy controller. The used membership functions forthe input variable $e$,the input variable $\dot{e}$ and theoutput variable $u$ are, respectively, shown in the upper figure, middle figure and down figure of Fig. 4.

$$
\begin{aligned}
& \boldsymbol{R}^{1}: \text { If } e \text { is } Z \text {, then } u=Z, \\
& \boldsymbol{R}^{2}: \text { If } e \text { is } P \text { and } \dot{e} \text { is } P \text {, then } u=N N, \\
& \boldsymbol{R}^{3}: \text { If } e \text { is } P \text { and } \dot{e} \text { is } Z \text {, then } u=N, \\
& \boldsymbol{R}^{4}: \text { If } e \text { is } P \text { and } \dot{e} \text { is } N \text {, then } u=N^{-} \\
& \boldsymbol{R}^{5}: \text { If } e \text { is } N \text { and } \dot{e} \text { is } P \text {, then } u=P^{+}, \\
& \boldsymbol{R}^{6}: \text { If } e \text { is } N \text { and } \dot{e} \text { is } Z \text {, then } u=P, \\
& \boldsymbol{R}^{7}: \text { If } e \text { is } N \text { and } \dot{e} \text { is } N \text {, then } u=P P,
\end{aligned}
$$

Table 1. Fuzzy rules

\begin{tabular}{|c|c|c|c|}
\hline$\dot{e}$ & $\mathbf{P}$ & $\mathbf{Z}$ & $\mathrm{N}$ \\
\hline $\mathrm{P}$ & $\mathrm{NN}$ & $\mathbf{Z}$ & $\mathbf{P}^{+}$ \\
\hline $\mathrm{Z}$ & $\mathrm{N}$ & $\mathrm{Z}$ & $\mathrm{P}$ \\
\hline $\mathrm{N}$ & $\mathbf{N}^{-}$ & $\mathbf{Z}$ & $\mathrm{PP}$ \\
\hline
\end{tabular}

Figure 5 shows the scheme for fuzzy logic control of the cascade pathway. The two subsystem blocks with $\mathrm{x} 4$ input node and $\mathrm{x} 1, \mathrm{x} 2, \mathrm{x} 3$ output nodes are the cascade pathway system block. Figure 6 is the detailed signal flow of this block. In Fig. 5 the upper figure denoted by dashed red line simulates the dynamic behavior of the system with an exponential input (original systems). The down figure runs the dynamic behavior of the closed-loop fuzzy-logic controlled system. The experimental condition is set at $r=$ rOe-0.2twhere the nominal value of the independent variable is $x_{4}=r_{0}=3$. At this case $\left(x_{4}=3\right)$,the target steady state vales are estimated through Eq. (4): $\bar{x}=[15.48,29.86,5.185]^{T}$. In Fig. 5, the "Interpreted MATLAB Fcn" block (the bottom box in the middle) carries out steady state estimation. For clear comparison, the target values and the simulation results are displayed in the lower right corner of Fig. 5 and the target values is marked by a dashed blue block. The results show that the proposed controller is able to perfectly regulate the system back to the target steady state.

The Rule Viewer in fuzzy logic control subsystem supports peopleto real-time view the inference process. Figure 7 is the final result shown in the Rule Viewer. The estimated error $e_{2}=x_{2}-\bar{x}_{2}=0.0168$, the estimated error derivative $\dot{e}_{2}=\dot{x}_{2}-\bar{x}_{2}=-0.00272$ and the estimated independent variable $x_{4}=u(t)=3$ which equal to the nominal value $\left(r_{0}=3\right)$. Figure 8 shows the dynamic behavior of the system states $x_{1}$ (purple), $x_{2}$ (red)and $x_{3}$ (blue). The states of the original system are denoted as dashed lines, that of the controlled system are denoted as solid lines and green solid lines show the target values. The controlled system reaches the target at around 22.5 seconds.
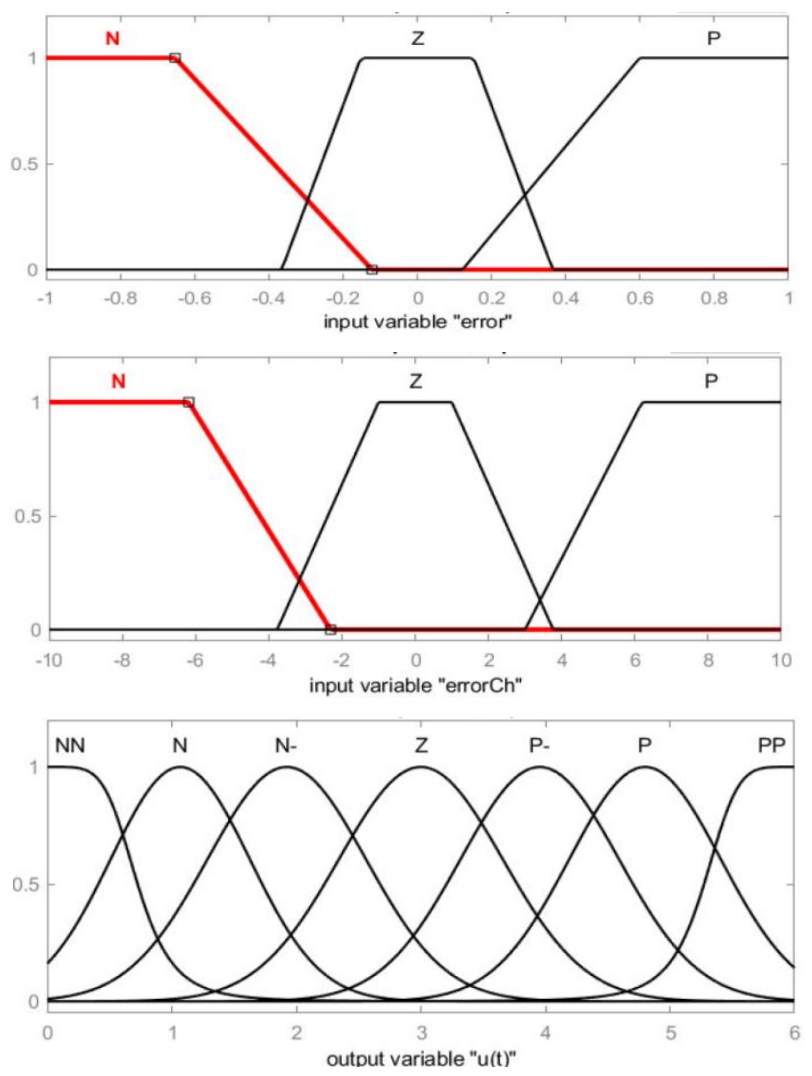

Fig4:Membership functionsfor the input variable $e$ (upper figure), the input variable $\dot{e}$ (middle figure) and the output variableu(down figure).
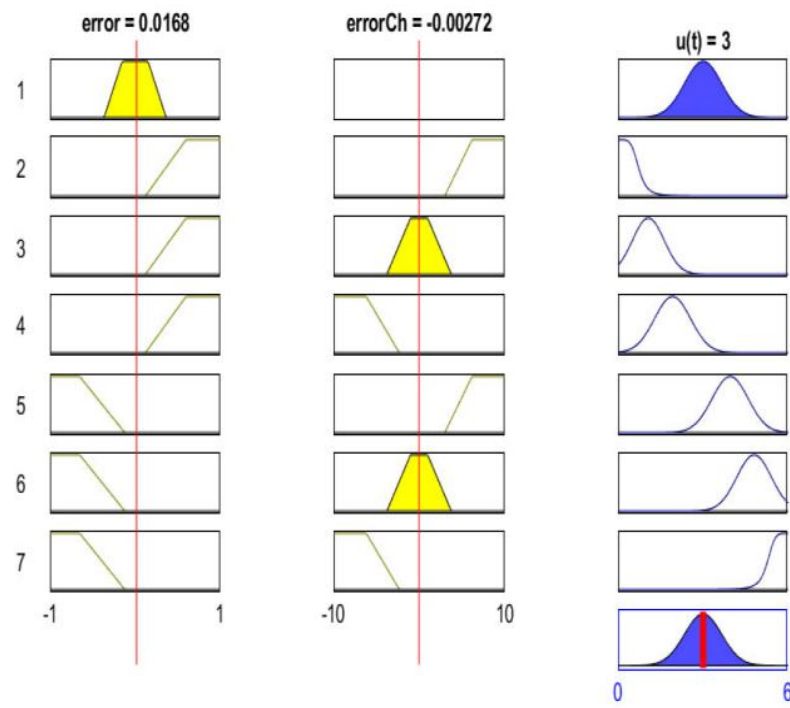

Fig 7:The final results of the error e, the error derivative $\dot{e}$ and the estimated system input $\mathrm{u}(\mathrm{t})$ (the independent variable $x_{4}$ ) shown in the Rule Viewer window.

Additional experiments are conducted at $r_{0}=0.75,1,1.5$, 2.The simulation results for these four experiments are shown in Table 2. The detailed results are shown in the supplemental file. The time for the controlled system to reach the target values is around 6 seconds, 7 seconds, 11 seconds and 15 seconds for $r_{0}=0.75,1,1.5,2$, respectively. All of the simulation results show that the proposed fuzzy logic controllers are able to force the system back to nominal steady states. The bigger the nonmail value of the independent variable is the longer the time to reach the target becomes. 


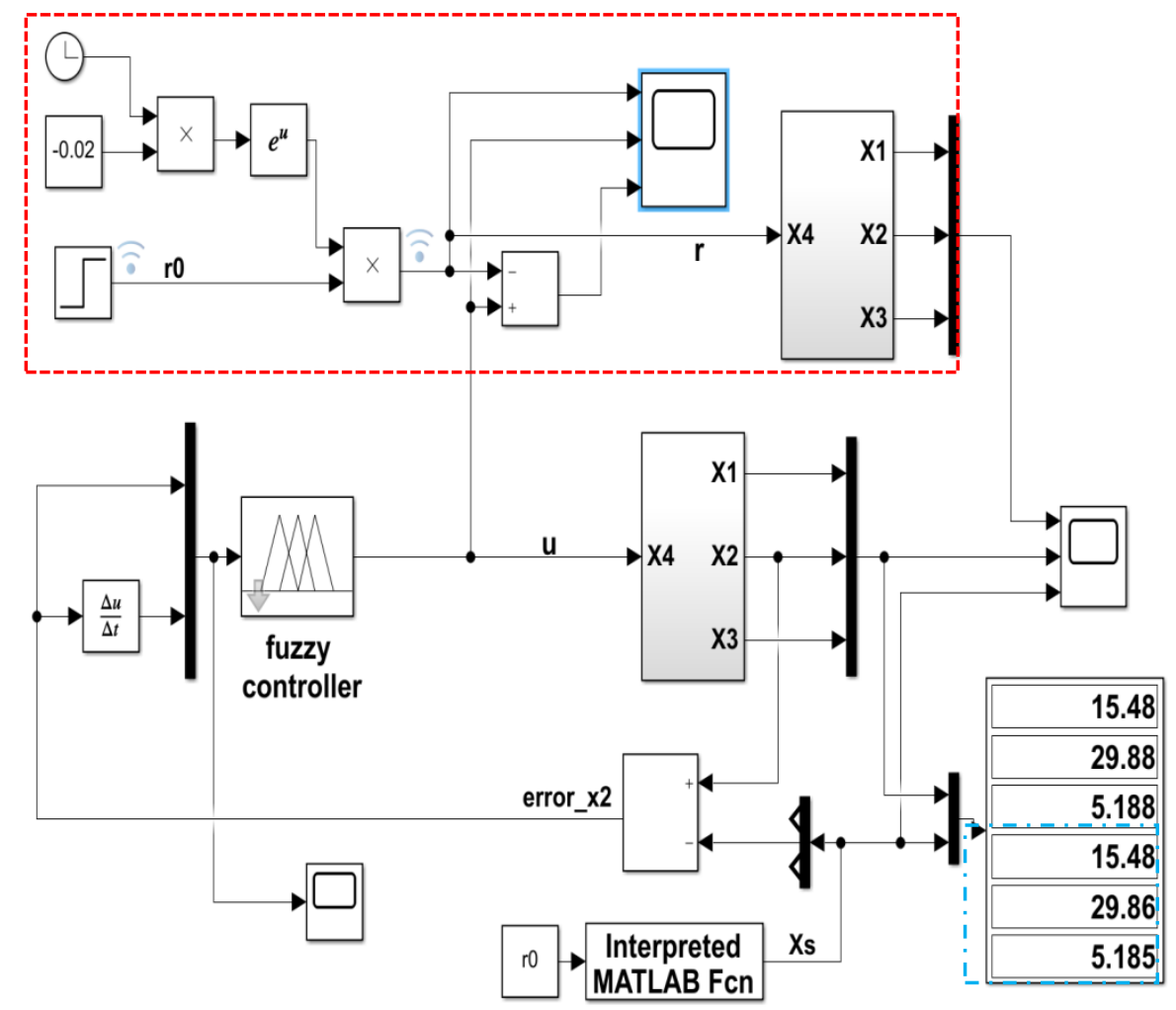

Fig5: Scheme for fuzzy Logic Control of the cascade pathway in Eq. (3). The right down table shows the estimated values (upper three rows) and the target values (down three rows)

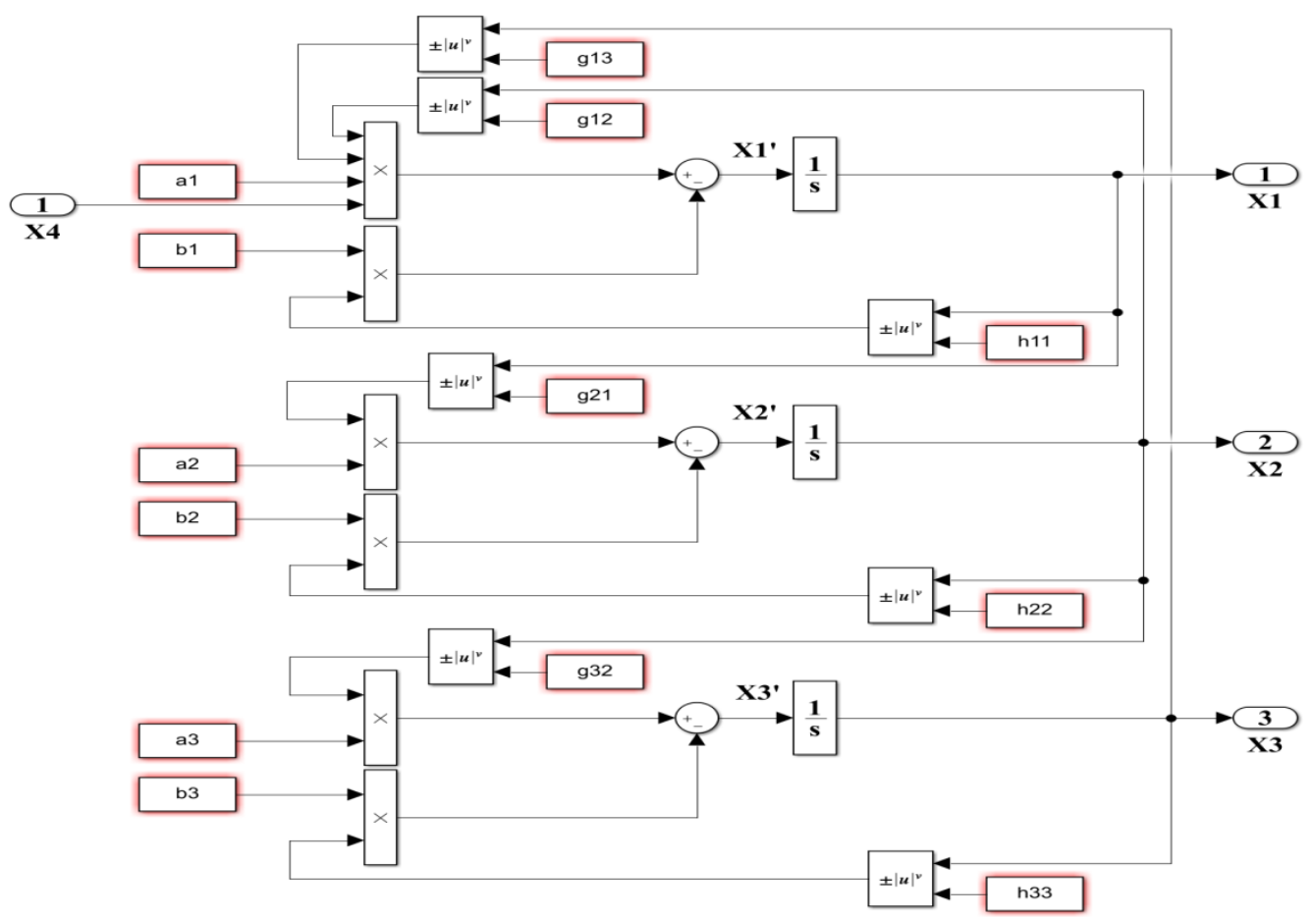

Fig 6: Block diagrams of the cascade system in Eq. (3). 


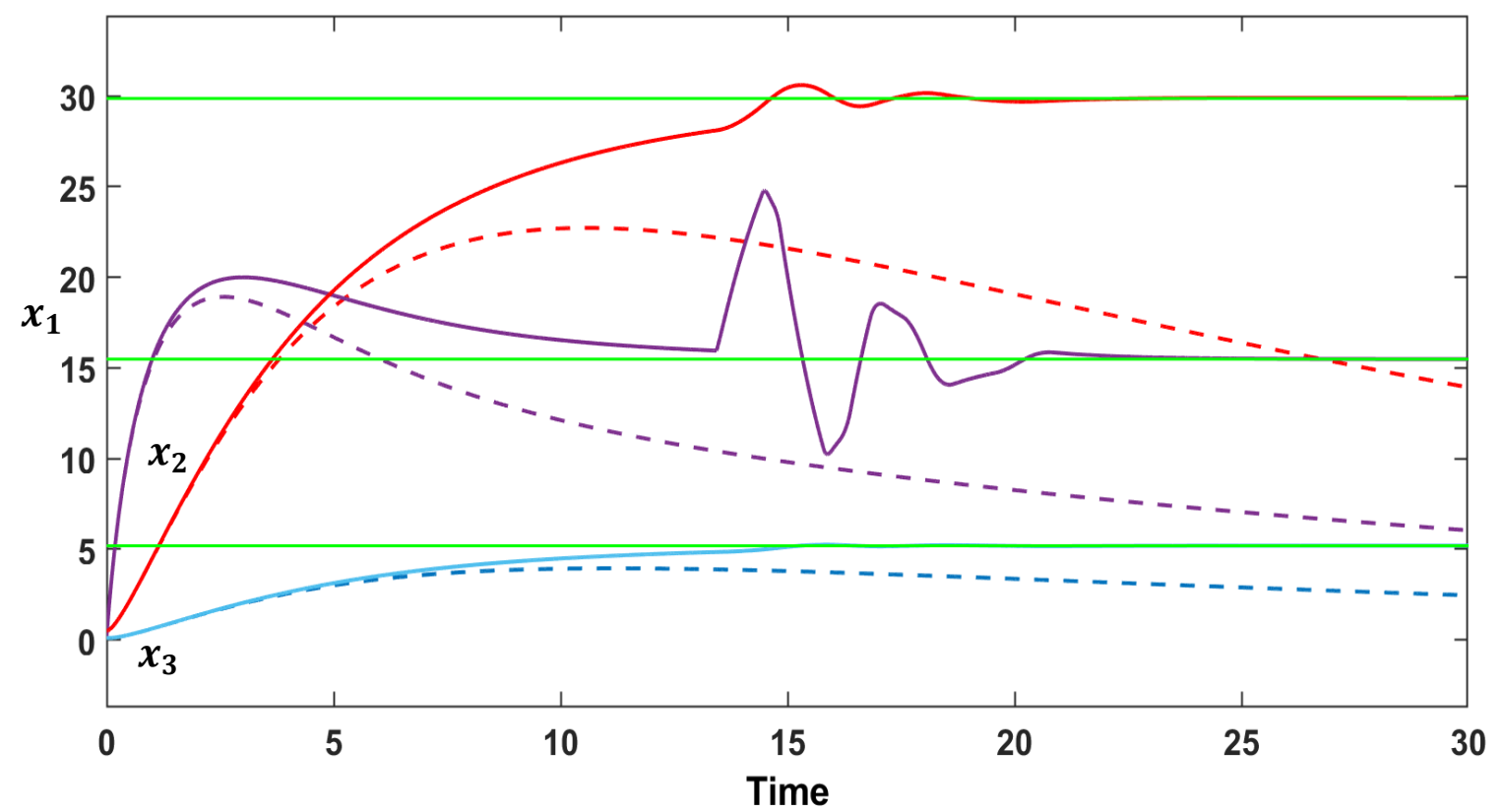

Fig 8: The dynamic behavior system states $x_{1}$ (purple), $x_{2}$ (red)and $x_{3}$ (blue). The states for the original system are denoted as dashed lines, that for the controlled system are denoted as solid lines and the target states are shown in green solid lines.

Table 2. Simulation results for $x_{4}=r_{0} e^{-0.2 t}$ with $r_{0}=0.75,1,1.5,2,3$.

\begin{tabular}{|c|c|c|c|c|c|}
\hline & \multicolumn{5}{|c|}{$x_{4}=\boldsymbol{r}_{\mathbf{0}} e^{-0.2 t}$} \\
\cline { 2 - 6 } & $\mathbf{0 . 7 5} e^{-0.2 t}$ & $\mathbf{1} e^{-0.2 t}$ & $\mathbf{1 . 5} e^{-0.2 t}$ & $\mathbf{2} e^{-0.2 t}$ & $\mathbf{3} e^{-0.2 t}$ \\
\hline estimated input $\mathrm{u}(\mathrm{t})$ & 0.75 & 1 & 1.5 & 2 & 3 \\
\hline regulating time $t_{s}$ & 6 & 7 & 11 & 15 & 22.5 \\
\hline target states $\bar{X}$ & {$\left[\begin{array}{c}1.835 \\
3.539 \\
0.6144\end{array}\right]$} & {$\left[\begin{array}{c}2.856 \\
5.509 \\
0.9565\end{array}\right]$} & {$\left[\begin{array}{c}5.329 \\
10.28 \\
1.785\end{array}\right]$} & {$\left[\begin{array}{c}8.296 \\
16 \\
2.778\end{array}\right]$} & {$\left[\begin{array}{c}15.48 \\
29.86 \\
5.185\end{array}\right]$} \\
\hline estimated states $X_{e s}$ & {$\left[\begin{array}{c}1.835 \\
3.539 \\
0.6144\end{array}\right]$} & {$\left[\begin{array}{c}2.856 \\
5.509 \\
0.9565\end{array}\right]$} & {$\left[\begin{array}{l}5.329 \\
10.28 \\
1.785\end{array}\right]$} & {$\left[\begin{array}{c}8.296 \\
16 \\
2.779\end{array}\right]$} & {$\left[\begin{array}{c}15.48 \\
29.88 \\
5.188\end{array}\right]$} \\
\hline error $e$ & -8.34 & -0.000373 & 0.00396 & 0.00124 & 0.0168 \\
\hline error derivative $\dot{\boldsymbol{e}}$ & $4.63 \times 10^{-6}$ & 0.000166 & -0.00128 & -0.00034 & -0.00272 \\
\hline
\end{tabular}

\section{CONCLUSION}

The degradation of biological functions changes the environment of organismsand as a result, organism loses regulation ability.For S-type biological systems, modellable experimental environments are denoted as independent variables, the values of which remain constant throughout an experiment. Time-varying independent variables are concerned to describe this kind of degradation phenomenon. A fuzzy logic controller is then proposed to regulate systems to target states. and estimate the nominal values of independent variables. Simulation results show that the proposed controller is able to not only successfully force systems to targetstates but also find out the nominal values of independent variables. This research initiatesthe application of fuzzy logic control in S-type biological systems. Follow-up research will apply fuzzy logic control to systems with generalized Michaelis-Menten kinetics, and develop various fuzzy compensators or fuzzy estimators to deal with noisecontaminated biological systems.

\section{ACKNOWLEDGMENTS}

This research was supported by grant number MOST 1092221-E-212-003 from the Ministry of Science and Technology of Taiwan, R.O.C.

\section{REFERENCES}

[1] Tyson, J.J. 1991. Modeling the cell division cycle: cdc2 and cyclin interactions.Proc. Natl. Acad. Sci. USA 88:7328-7332.

[2] Tyson, J.J., Chen, K.C.,and Novak, B. 2003. Sniffers, buzzers, toggles and blinkers: dynamics of regulatory and signaling pathways in the cell. Curr. Opin. Cell Biol. 15:221-231.

[3] Tavassoly, I.,Parmar, J., Shajahan-Haq, A,, Clarke, R., Baumann, W., and Tyson, J.J. 2015. Dynamic modeling of the interaction between autophagy and apoptosis in mammalian cells.CPT Pharmacometrics Syst Pharmacol 
4(4):263-72.

[4] Liu, P. K., and Wang, F. S. 2010.Inverse problems of biological systems using multi-objective optimization. Journal of the Chinese Institute of Chemical Engineers. 39(5):399-406.

[5] Wu, S. J., Wu, C. T.,and Chang, J. Y. 2012. Fuzzy-based self-interactive multi-objective evolution optimization for reverse engineering of biological networks.IEEE Transactions on Fuzzy Systems20(5):865-882.

[6] Wu, S. J., Wu, C. T.,and Chang, J. Y. 2013.Adaptive neural-based fuzzy modeling for biological systems. Math Biosci 242(2):153-60.

[7] Wu, S. J., and Wu, C. T. 2013.Computational optimization for S-type biological systems: cockroach genetic algorithm.Math Biosci 245(2):299-313.

[8] Wu, S. J, and Wu, C. T. 2014.Seeding-inspired chemotaxis genetic algorithm for the inference of biological systems.Comput Biol Chem 53(2):292-307.

[9] $\mathrm{Wu}, \mathrm{S}$. J, and $\mathrm{Wu}$, C. T. 2015.A bio-inspired optimization for inferring interactive networks: cockroach swarm evolution. Expert Syst Appl 42(6): 3253-3267.

[10] Wu, S. J, and Wu, C. T.2018.Smarten up computational intelligence to decipher time series data.Appl Soft Comput72:442-456.

[11] Liu, S., Tao, C., Huang, Z, and Huang S. 2010. Modeling of p53 signaling pathway based on S-system equations.Journal of Biomedical Engineering 27(3):50510 (Chinese)

[12] Luo, Z.P., An, K.N. 2001.Fuzzy systems in biomedical science. BIOMEDICAL SCIENCE. International Journal of General Systems 30(2):209-217.

[13] Komiyama, M., Yoshimoto, K., Sisido, M., Ariga, K. 2017. Chemistry can make strict and fuzzy controls for bio-Systems: DNA nanoarchitectonics and cell- macromolecular nanoarchitectonics. Bulletin of the Chemical Society of Japan 90(9):967-1004.

[14] Abyad, M., Karama, A., and Khallouq, A. 2017.Modelling and control of a biological process using the fuzzy logic Takagi-Sugeno. In Proceeding of the 2017 International Renewable and Sustainable Energy Conference (IRSEC).

[15] Bordon, J., Moskon, M., Zimic, N., and Mraz, M. 2015. Fuzzy logic as a computational tool for quantitative modelling of biological systems with uncertain kinetic data. IEEE/ACM Transactions on Computational Biology and Bioinformatics 12(5):1199-1205.

[16] Liu, F., Heiner, M., and Gilbert, D.2020.Fuzzy Petri nets for modelling of uncertain biological systems. Briefings in Bioinformatics 21(1):198-210.

[17] Liu, F., Sun, W., Heiner, H., and Gilbert, G. 2021. Hybrid modelling of biological systems using fuzzy continuous Petri nets. Briefings in Bioinformatics22(1):438-450.

[18] Zhu, X.L., Jiang, Z.Y., Wang, B., and He Y.J. 2018. Decoupling control based on fuzzy neural-network inverse system in marine biological enzyme fermentation process. IEEE Access, 6:36168-36175.

[19] Wu, S.J., Wu,C.T., and Chang J.Y.2013.Adaptive neuralbased fuzzy modeling for biological systems. Math Biosci242(2):153-160.

[20] http://www.medicineslearningportal.org/2015/07/kidneyand-liver-clearance.html

[21] Dyken, J. D. V. 2017. Noise slows the rate of MichaelisMenten reaction. J Theor Biol 430:21-31.

[22] Tsai, K.Y., Wang, F.S. 2005.Evolutionary optimization with data collocation for reverse engineering of biological networks. Bioinformatics 21(7):1180-8. 